\title{
SAÚDE MASCULINA: O PARALELO PREVENÇÃO X CUIDADO À LUZ DA TEORIA DA AÇÃO PLANEJADA (TAP)
}

\section{Jéssica Bazilio Chaves}

Mestre em Psicologia pela Universidade Federal de Alagoas (UFAL). Terapeuta Ocupacional pela Universidade Estadual de Ciências da Saúde de Alagoas (UNCISAL), Brasil.

\section{Maria Lúcia Vicente da Silva}

Graduanda em Psicologia pela Universidade Federal

\section{Daniela Santos Bezerra}

Graduada em Psicologia pela Universidade Federal de Alagoas (UFAL), Brasil.

\section{Sheyla C. S. Fernandes}

Doutora em Psicologia Social pela Universidade Federal da Bahia (UFBA). Docente adjunta da Universidade Federal de Alagoas (UFAL), Brasil.

\section{Emanuel Duarte de Almeida Cordeiro}

Doutorando no Programa de Psicologia Cognitiva pela Universidade Federal de Pernambuco (UFPE), Brasil. de Alagoas (UFAL), Brasil.

RESUMO: Este estudo teve como objetivo avaliar a intenção de homens, entre 25 e 59 anos, em um distrito sanitário de Maceió (AL), por procurar a Unidade Básica de Saúde (UBS). Realizaram-se duas etapas: (1) análise das crenças de uma parcela da amostra, para a confecção de um instrumento quantitativo ( $\mathrm{N}=25)$; (2) aplicação desse instrumento em uma amostra maior dessa população $(\mathrm{N}=106)$. Os resultados demonstraram que a intenção dos homens em procurar a UBS está associada aos atributos que facilitam e/ou dificultam a realização desse comportamento. Concluiu-se que apenas o construto Percepção de Controle se relacionou com a Intenção Comportamental. No entanto, os construtos Norma Subjetiva e Atitude não apresentaram impacto significativo sobre a Intenção dos homens de procurar a UBS. Discutiu-se a importância da elaboração de políticas públicas mais eficientes para o cuidado da saúde masculina, bem como a relevância de evidências empíricas para reflexões mais consistentes nessa área.

PALAVRAS-CHAVE: Saúde do homem; Atitude; Atenção primária à saúde.

\section{MALE HEALTH: PREVENTION VERSUS CARE IN THE LIGHT OF PLANNED ACTIVIT Y THEORY}

ABSTRACT: Current analysis evaluates why males, aged between 25 and 59 years, apply to a Basic Health Unit (UBS), in a health district unit in Maceió AL Brazil. Two stages were undertaken: (1) analysis of beliefs of a part of the sample for the elaboration of a qualitative instrument $(\mathrm{N}=25)$; (2) the application of the instrument to a larger sample of the population $(\mathrm{N}=106)$. Results demonstrate that the reason why males go to UBS is associated with attributes that facilitate or make difficult such behavior. Results show that only the construct Control Perception is related to the Behavior Intention. However, the construct Subjective Norm and Attitude did not have any significant impact on male intention for going to UBS. More efficient public policies should be prepared for male health and for the relevance of empirical evidence for more consistent conclusions on the theme.

KEY WORDS: Men's Health; Attitude; Primary Health Care.

\section{INTRODUÇÃO}

O modo de socialização dos homens tem sido apontado como

Autor correspondente

Jéssica Bazilio Chaves

E-mail: jessicabazilioc@gmail.com fator negativo em relação aos cuidados de saúde, influenciando de modo direto na procura pelos serviços de saúde, sobretudo os da rede 
de atenção primária, que atuam na promoção da saúde e prevenção de doenças. A falta de preocupação do homem com sua saúde está, portanto, refletida nos índices de morbimortalidade que apontam disparidades quando comparados à população feminina ${ }^{1}$.

Os altos índices de morbimortalidade masculina ${ }^{2}$ levam à adoção da concepção relacional de gênero, como forma de interpretar os processos de saúde e doença que afetam o universo masculino ${ }^{3-4}$. Os homens são mais propensos e apresentam maior vulnerabilidade a adquirir doenças que as mulheres. Isso se deve a uma exposição elevada às condições de risco culturais e comportamentais, atravessadas por estereótipos de gênero, que não valorizam práticas de prevenção e cuidados de saúde. A não procura pelos serviços de saúde resulta em homens mais vulneráveis a lesões 5 .

Souza, Queiroz, Florencio, Portela, Fernandes e Pereira $^{6}$ apontam que os homens entrevistados em seu estudo consideraram o cuidado como um elemento essencialmente feminino e adotavam o modelo dominante de masculinidade, distanciando-se das condutas de autocuidado. Os participantes afirmam que as mulheres procuram os serviços de saúde com mais frequência e por precaução porque se vinculam ao ambiente doméstico, enquanto que o homem faz parte do mundo do trabalho. Deste modo, elas teriam mais disponibilidade para o autocuidado.

Schraiber ${ }^{7}$ verificou a relevância envolvida nessa distinção entre as necessidades das mulheres e dos homens. A autora enfatiza que as diferenças entre ambos os sexos não devem implicar necessariamente em práticas profissionais desiguais, baseadas na cultura tradicional de gênero. Essa concepção enfatiza que a prática profissional deve exigir maior atenção quanto às necessidades dos homens e às práticas de saúde.

Diante de tal contexto, baseada nos preceitos do Sistema Único de Saúde (SUS), a Atenção Primária à Saúde (APS) tem como principal foco a Estratégia de Saúde da Família (ESF), buscando ser a porta de entrada para os serviços de saúde, o contato preferencial dos usuários no sistema, além de resolver os problemas de saúde de maior frequência e relevância em seu território ${ }^{8}$. Considerando esse quadro e objetivando a melhoria das condições de saúde da população masculina no Brasil, a Política Nacional de Atenção Integral à Saúde do Homem (PNAISH) foi criada em 2009 pelo Ministério da Saúde. De modo geral, essa política apresenta como proposta contribuir para a diminuição da morbidade e mortalidade dessa população, a fim de promover ações de saúde significativas para o entendimento da realidade masculina nos seus diversos cenários socioculturais e político-econômicos $^{9-10}$.

A PNAISH apresenta como enfoque metodológico a assistência aos homens entre 25 e 59 anos de idade, faixa etária que compõe a parcela da força produtiva e que nunca fez parte de qualquer política de saúde 9. Considerando que os serviços de saúde são mais acessados por crianças, mulheres e idosos, objetiva-se alcançar essa outra parte da população, a masculina, que apresenta os maiores indicadores de não aderência a tais serviços $^{11}$.

O Ministério da Saúde 9 acredita que compreendendo as barreiras socioculturais e institucionais é possível propor estratégias que promovam o acesso dos homens aos serviços de atenção primária, que devem ser a porta de entrada ao sistema de saúde, a fim de resguardar a prevenção e a promoção como eixos necessários e fundamentais de intervenção.

Entre as áreas da ciência que estudam fenômenos comportamentais e de saúde encontra-se a psicologia social, em que é comum o estudo do comportamento referente à mudança para promoção de hábitos saudáveis e aderência terapêutica ${ }^{12}$. Moutinho e Roazzi ${ }^{12}$ argumentam que a Teoria da Ação Planejada (TAP) é um modelo bastante utilizado em pesquisas não só na área da psicologia, como também em outras áreas que se preocupam com a adoção de comportamentos preventivos em saúde. Isso se deve à possibilidade de conhecimento das intenções de um dado comportamento em uma determinada população, permitindo aos programas e políticas de saúde a utilização de estratégias mais eficazes e resolutivas.

A TAP ${ }^{13}$ foi projetada a fim de prever e explicar o comportamento humano em determinados contextos. A TAP é uma extensão da Teoria da Ação Racional (TAR) criada na década de 1960 por Martin Fishbein e revisada e aprofundada por Fishbein e Ajzen na década de $1970^{13}$ - que se mostrou necessária pela limitação do modelo 
original da TAR em lidar com comportamentos sobre os quais os indivíduos apresentam controle volitivo incompleto $^{14}$.

Assim como na TAR, a intenção do indivíduo de realizar um determinado comportamento é um fator central na TAP. As intenções apontam os fatores motivacionais que influenciam um comportamento, indicando de que forma os sujeitos estão dispostos a tentar e o quanto de esforço pretendem demandar em sua realização. De modo geral, quanto mais forte for a intenção de aderir a um comportamento, maior será a probabilidade de sua execução ${ }^{14}$.

A base da TAR é formada pelas crenças e estas são compreendidas como implícitas às Atitudes e às Normas Subjetivas, o que as situam como determinantes de intenções e comportamentos ${ }^{12}$. De modo geral, as crenças correspondem à informação que o sujeito possui a respeito de um determinado objeto, agregando a ele alguma característica.

Em contrapartida, o modelo da TAR exibiu limitações, tendo em vista que desconsiderava os comportamentos que não se encontravam sob o domínio volitivo ou motivacional dos indivíduos, conseguindo então o comportamento ser instigado também por fatores como os hábitos. Desse modo, Ajzen buscou a ampliar e expandir a TAR por meio da TAP ${ }^{14,12}$.

Com relação a esse novo modelo, além do que apontava a TAR, tem sido incorporado outro componente de previsão: a percepção de controle. Esta é definida como a percepção acerca da presença de fatores que podem facilitar ou impedir a execução do comportamento. Considera-se que a maior percepção de controle do comportamento coincide com a maior probabilidade de que o desempenho do comportamento tenha êxito ${ }^{14}$.

A atitude, por sua vez, é considerada como uma tendência para responder de maneira preferencial, ou seja, o quanto se é favorável ou desfavorável perante o comportamento analisado. Trata-se da perspectiva subjetiva de que o comportamento fornecerá algum efeito, o juízo pessoal sobre o comportamento com base nas consequências ${ }^{14}$. Em relação à norma subjetiva, refere-se à percepção do indivíduo quanto à pressão social exercida sobre o mesmo para que execute ou não o comportamento em questão. São as influências sobre o comportamento das pessoas, provenientes da pressão social de pais, grupos de referência, cultura, opinião pública ou instituições ${ }^{14}$.

Como regra geral, quanto mais favorável a atitude e a norma subjetiva em relação a um comportamento, e quanto maior o controle comportamental percebido, mais forte deve ser a intenção de um indivíduo realizar o comportamento em consideração ${ }^{15}$. Sendo assim, como buscamos analisar a disposição dos homens para procurar as Unidades Básicas de Saúde (UBS), tornou-se válido empregar o modelo da TAP para assim conhecer as crenças e, por conseguinte, predizer a intenção para efetuar o comportamento em questão.

É com base nesses conceitos e recomendações que se segue este estudo, com o objetivo de 1) analisar as crenças comportamentais masculinas acerca de procurar a UBS; 2) elaborar um instrumento para a avaliação da intenção masculina de procurar a UBS; 3) analisar em que medida as atitudes, as normas subjetivas e a percepção de controle estão associadas à intenção comportamental de procurar a UBS.

\section{METODOLOGIA}

\section{ELABORAÇÃO DO INSTRUMENTO}

\section{Primeira etapa - levantamento das crenças}

Conforme a metodologia utilizada por Ajzen e Fishbein $^{13}$, a construção do instrumento foi efetivada por meio da análise de crenças e atitudes junto a um grupo de 25 homens, cadastrados em uma das cinco UBS de um distrito sanitário de Maceió (Alagoas), com idades entre 25 e 59 anos $(M=42,56 ; \mathrm{DP}=10,80)$, seguindo o critério do público-alvo da PNAISH ${ }^{9}$. Os participantes indicaram estar casados (68\%), ter filhos (88\%) e exercer trabalho informal (72\%). Apesar de alfabetizados (76\%), muitos não acessaram o ensino formal (40\%) e apresentavam primeiro grau incompleto (80\%).

As entrevistas semiestruturadas foram realizadas individualmente em domicílios, espaços comunitários e nas UBS para maior comodidade dos participantes, 
sendo utilizado o gravador para o registro das falas. Os participantes foram solicitados a responder sobre as vantagens de procurar a UBS, sobre quais as pessoas cujas opiniões sobre a realização desse comportamento seriam importantes e os fatores que facilitariam ou dificultariam a procura à UBS anualmente. As vantagens $\mathrm{e}$ desvantagens são consideradas crenças comportamentais. As pessoas ou entidades mais citadas pelos respondentes são consideradas como referentes ligados às crenças normativas. Os fatores que facilitam ou dificultam a procura pela unidade básica estão relacionados às crenças de controle.

Os discursos foram organizados e analisados por meio da análise de conteúdo de $\operatorname{Bardin}^{16}$, tendo sido executadas as etapas: a) transcrição de todas as crenças e referentes emitidos pela amostra, b) leitura flutuante, c) recorte das Unidades de Contexto Elementar (UCEs), d) agrupamento das UCEs de significado semelhante, e) organização das UCEs de significado semelhante em categorias, subcategorias e unidades temáticas (contribuição de 03 juízes - 01 doutor e 02 alunos de pós-graduação), e f) contagem da frequência por subcategoria, adotando o critério da frequência mínima, cujo valor deve ser igual ou superior a $75 \%$, com que cada Crença Comportamental, de Controle e Normativa foi eliciada para o comportamento em questão.

Foram obtidas 47 crenças comportamentais acerca de procurar a UBS, de um total de 52 crenças emitidas. Estas foram organizadas em oito dimensões: (1) cuidar da saúde; (2) não ter atendimento; (3) acolhimento dos profissionais; (4) atendimento com atraso; (5) tratamento de doenças; (6) atendimento próximo de casa; (7) faltar medicação e (8) não vê vantagem. Como principais referentes foram encontrados: a esposa, a família, os filhos, irmãs e si mesmo. Por fim, em relação às crenças de controle, foi obtido um total de 25 crenças de controle modais salientes, de um total de 39 crenças emitidas. Dessas crenças, surgiram quatro subcategorias: (1) tomar medicação por obrigação; (2) não ter vaga para atendimento; (3) horário de trabalho e (4) estar doente.

\section{Segunda etapa - construção do instrumento}

Após a identificação e agrupamento das "crenças salientes", foi dado prosseguimento à pesquisa por meio da elaboração de um instrumento quantitativo. Os itens construídos para avaliar as crenças, assim como aqueles envolvendo atitude, norma subjetiva e intenção, foram elaborados a partir das instruções dos autores ${ }^{13}$ acerca da forma das escalas, todas de sete pontos, e da redação dos itens para as diversas variáveis do modelo.

\section{PARTICIPANTES DO ESTUDO}

A amostra do estudo foi composta por 106 participantes cadastrados em três das cinco UBS de um distrito sanitário de Maceió, Alagoas (sendo 27 participantes cadastrados em uma primeira UBS, 30 cadastrados em uma segunda UBS e 49 em uma terceira UBS). Todos os participantes eram homens entre $25 \mathrm{e}$ 59 anos $(M=43,10 ; D P=10,86)$, seguindo o critério do público-alvo da PNAISH ${ }^{9}$. Em sua maioria, casados (47,2\%), com ensino médio incompleto $(31,15 \%)$ e renda familiar de dois salários mínimos (34\%). Com relação ao perfil epidemiológico, identificou-se um considerável índice de hipertensos (23,6\%), fumantes $(21,7 \%)$, alcoolistas (17\%) e diabéticos (14,2\%).

\section{INSTRUMENTOS}

Foi aplicado um questionário quantitativo construído a partir das crenças salientes obtidas na primeira etapa do estudo, totalizado em 32 itens, sendo 28 alocados como variáveis independentes (VI) e quatro como variáveis dependentes (VD). "Atitude" com dez itens $(5$ itens referentes às Crenças Comportamentais e 5 itens referentes à Avaliação das Consequências); "Norma Subjetiva" com dez itens (5 itens referentes às Crenças Normativas e 5 itens referentes à Motivação para Concordar com o referente) e "Percepção de Controle" com oito itens ( 4 itens referentes às Crenças de Controle e 4 referentes à Habilidade para Controlar a Situação), representam as VIs. Por fim, quatro itens acerca da "Intenção Comportamental", representando as VDs. A escala de respostas utilizada para medir as crenças que influenciam a intenção de procurar a UBS foi do tipo Likert, variando de 1 a 7 , desde "Discordo totalmente a "Concordo totalmente", "Muito a Pouco" e "Pouco importante a Muito importante", com ponto médio igual a 4 . 


\section{PROCEDIMENTOS}

Os participantes do estudo foram contatados a partir dos agentes comunitários de saúde do distrito sanitário em questão, que acompanharam a aproximação às comunidades e domicílios, a fim de encontrar os homens que atendessem aos critérios do estudo. Os critérios de exclusão foram recusar-se a participar da pesquisa, não estar em condições para responder as perguntas, não estar na faixa etária dos 25 a 59 anos e não estar cadastrado em alguma UBS do distrito sanitário de Maceió.

Todos os participantes foram voluntários e assinaram o Termo de Consentimento Livre e Esclarecido, indicando a autorização e o entendimento de todos os aspectos de investigação da pesquisa, de acordo com a resolução n ${ }^{0} 466 / 12$ do Conselho Nacional de Saúde (Ministério da Saúde, 2012). O projeto foi aprovado pelo Comitê de Ética em Pesquisa da Universidade Federal de Alagoas (UFAL) (parecerCAAE 43079214.8.0000.5013).Em seguida, foi aplicado o questionário que contém os itens referentes às variáveis da TAP. Considerando que grande parte do grupo de respondentes era composto de pessoas analfabetas funcionais, os instrumentos foram aplicados individualmente nos domicílios, espaços comunitários e nas UBS's. A coleta de dados foi realizada no período de julho a novembro de 2015. O tempo médio da aplicação do instrumento foi de 10 minutos.

\section{ANÁLISE DOS DADOS}

Os dados foram tabulados no SPSS 23.0 (Statistical Package for Social Sciences), onde foram realizadas análises descritivas (frequência, porcentagem, média, desvio-padrão) para a caracterização dos participantes, além de análises fatoriais exploratórias e análises de confiabilidade interna para avaliação das variáveis. Para verificar as relações existentes entre todas as variáveis independentes que compõem o modelo teórico e a variável dependente, verificando a força e o tipo dessas relações, utilizou-se o coeficiente de correlação $r$ de Pearson e a Análise de Regressão Múltipla (hierárquica).

\section{RESULTADO}

Para analisar os resultados, inicialmente foi realizada uma Análise Fatorial Exploratória (AFE) dos componentes principais (rotação Varimax) de cada construto do instrumento com o objetivo de averiguar a sua configuração. Para verificar se os dados poderiam ser submetidos ao processo da AFE, foi utilizado o critério de Kaiser-Meyer-Olkin (KMO) e o Teste de Esfericidade de Bartlett que testa a existência de não correlação entre os dados, em que os níveis de significância devem ser $\mathrm{p}<0,05$. Consideram-se, também, os eigenvalues e a variância explicada de cada variável ${ }^{17}$.

$\mathrm{O}$ construto Atitude, referente às Crenças Comportamentais (CC) e Avaliação das Consequências (AC) foi reduzido a um único fator, composto por quatro itens, explicando $63,847 \%$ das atitudes em relação a procurar a UBS $(\mathrm{KMO}=0,644 ; \mathrm{X} 2=176,822 ; \mathrm{p}=0,000)$ (Tabela 1).

Tabela 1. Análise Fatorial do construto Atitude

\begin{tabular}{|c|c|}
\hline Itens & \\
\hline 15- Cuidar da saúde é importante & ,908 \\
\hline $\begin{array}{l}\text { 16- Ser acolhido pelos profissionais de saúde é } \\
\text { importante }\end{array}$ &, 888 \\
\hline $\begin{array}{l}\text { 18- Ter tratamento quando estiver doente é } \\
\text { importante }\end{array}$ &, 665 \\
\hline $\begin{array}{l}\text { 19- Ter atendimento de saúde perto de casa é } \\
\text { importante }\end{array}$ &, 707 \\
\hline KMO & ,691 \\
\hline Eigenvalue (valor próprio) & 2,554 \\
\hline Variância explicada & 63,847 \\
\hline Alfa de Cronbach & 0,80 \\
\hline
\end{tabular}

O construto Norma Subjetiva, estruturado pelas Crenças Normativas (CN) e Motivação para Concordar com o Referente (MCR), foi reduzido a uma dimensão composta por quatro itens, explicando $54,186 \%$ de sua variância $(\mathrm{KMO}=0,508 ; \mathrm{X} 2=481,644 ; \mathrm{p}=0,000)$ (Tabela 2). 
Tabela 2. Análise Fatorial do construto Norma Subjetiva

\begin{tabular}{lc}
\hline \multicolumn{1}{c}{ Itens } & \\
\hline $\begin{array}{l}\text { 6- Minha família acha que eu devo procurar o posto } \\
\text { de saúde }\end{array}$ &, 603 \\
$\begin{array}{l}\text { 7- As mulheres da minha família acham que eu devo } \\
\text { procurar o posto de saúde }\end{array}$ &, 702 \\
$\begin{array}{l}\text { 25- O quanto você se importa com a opinião de sua } \\
\text { família? }\end{array}$ &, 824 \\
$\begin{array}{l}\text { 26- O quanto você se importa com a opinião das } \\
\text { mulheres de sua família? }\end{array}$ &, 791 \\
\hline KMO &, 508 \\
Eigenvalue (valor próprio) & 2,167 \\
Variância explicada & 54,186 \\
Alfa de Cronbach & 0,72 \\
\hline
\end{tabular}

O construto Percepção de Controle, que tem como base as Crenças de Controle (CCR) e Habilidade para Controlar a Situação (HCS), foi reduzido a uma dimensão constituída por três itens que explicam $56,085 \%$ da variância $(\mathrm{KMO}=0,503 ;$ X $2=48,858 ; \mathrm{p}=0,000)$ (Tabela 3).

Tabela 3. Análise Fatorial do construto Percepção de Controle

\begin{tabular}{lc}
\hline \multicolumn{1}{c}{ Itens } & Carga fatorial \\
\hline $\begin{array}{l}\text { 21- Ter vaga para ser atendido no posto de } \\
\text { saúde é }\end{array}$ &, 519 \\
22- Estar doente e procurar o posto de saúde é &, 883 \\
23- Precisar tomar medicação no posto de &, 795 \\
saúde é &, 503 \\
\hline KMO & 1,683 \\
Eigenvalue (valor próprio) & 56,085 \\
Variância explicada & 0,60 \\
Alfa de Cronbach & \\
\hline
\end{tabular}

Por fim, o construto Intenção Comportamental foi reduzido a três itens dispostos em um único fator, explicando 59,939\% de sua variância $(\mathrm{KMO}=0,585$; X 2 $=52,918 ; \mathrm{p}=0,000)($ Tabela 4$)$.
Tabela 4. Análise Fatorial do construto Intenção Comportamental

\begin{tabular}{lc}
\hline \multicolumn{1}{c}{ Itens } & $\begin{array}{c}\text { Carga } \\
\text { fatorial }\end{array}$ \\
\hline $\begin{array}{l}\text { 29-As pessoas cujas opiniões são importantes para } \\
\text { mim, apoiariam a procura pelo posto de saúde (nos } \\
\text { próximos 12 meses) }\end{array}$ &, 622 \\
$\begin{array}{l}\text { 31- Eu me esforçarei para procurar o posto de } \\
\text { saúde (nos próximos 12 meses) caso isso me traga } \\
\text { vantagens }\end{array}$ &, 862 \\
$\begin{array}{l}\text { 32- Eu planejo procurar o posto de saúde (nos } \\
\text { próximos 12 meses) se tiver condições para isso }\end{array}$ &, 818 \\
\hline KMO & 0,585 \\
Eigenvalue (valor próprio) & 1,884 \\
Variância explicada & 59,939 \\
Alfa de Cronbach & 0,67 \\
\hline
\end{tabular}

Foi adotada a análise do coeficiente de correlação de Pearson (r) para verificar as relações existentes entre os construtos Atitude, Norma Subjetiva, Percepção de Controle e Intenção Comportamental, a força e o tipo dessas relações. O teste apontou que apenas o construto Percepção de Controle se correlaciona positivamente com a Intenção Comportamental ( $r=0,353 ; p>0,01)$. De acordo com a TAP, a Percepção de Controle pode estar relacionada tanto por via indireta com o comportamento, por meio da Intenção Comportamental, como diretamente a ele (sem a mediação intencional $)^{13}$.

Entretanto, para a teoria, é preciso que a Atitude e a Norma Subjetiva combinadamente com a Percepção de Controle estejam correlacionadas com a Intenção Comportamental para que esta seja mais forte, o que não foi encontrado neste estudo.

Ademais, foi possível identificar uma correlação (apesar de baixa) entre os construtos Percepção de Controle e Atitude $(\mathrm{r}=0,201 ; \mathrm{p}>0,05)$. Isso pode significar que, para os homens estudados, o controle necessário para realizar o comportamento (procurar a UBS) está relativamente relacionado à avaliação positiva que os mesmos realizam a respeito das vantagens em efetivar o comportamento em questão.

Quanto à análise de predição de cada VI para a intenção de procurar a UBS, foi possível verificar por meio de uma Análise de Regressão Múltipla (hierárquica) um modelo estatisticamente significativo $[\mathrm{F}(1,99)=14,05$; 
$\left.\mathrm{p}<0,001 ; \mathrm{R}^{2}=0,12\right]$ que inclui apenas a Percepção de Controle como preditora da Intenção Comportamental de procurar a UBS $(\mathrm{B}=0,35 ; \mathrm{t}=3,74 ; \mathrm{p}<0,001)$, explicando $12 \%$ desta intenção (Tabela 5). Apesar da avaliação positiva (vantagens) de procurar a UBS estar relacionada à intenção em procurar a UBS, os resultados evidenciaram que é o controle comportamental percebido sobre a enfermidade, a consequente necessidade de ser medicado e a possibilidade de vagas para ser atendido que se constituem como elementos capazes de explicar uma parte dessa intenção.

Tabela 5. Análise de Regressão Múltipla

\begin{tabular}{lcccccc}
\hline \multicolumn{1}{c}{$\mathbf{V I}$} & $\mathbf{R}$ & $\mathbf{R}^{2}$ & $\mathbf{F}$ & Beta & $\mathbf{T}$ & $\mathbf{p}$ \\
\hline $\begin{array}{l}\text { Percepção } \\
\text { de Controle }\end{array}$ & 0,35 & 0,12 & 14,05 & 0,43 & 3,74 & 0,00 \\
\hline Atitudes & 0,35 & 0,12 & 7,04 & $-0,04$ & $-0,39$ & 0,69 \\
\hline $\begin{array}{l}\text { Norma } \\
\text { Subjetiva }\end{array}$ & 0,37 & 0,13 & 5,13 & 0,09 & 1,13 & 0,26 \\
\hline
\end{tabular}

Variável dependente: Intenção

Em relação às Atitudes e às Normas Subjetivas, é possível verificar que a capacidade preditiva desses construtos para a Intenção Comportamental não desempenha papel explicativo, contrariando o modelo proposto pela TAP. Nota-se que ter como importante o cuidado à saúde, o atendimento satisfatório e o incentivo da família para tais ações, não interferem significativamente na intenção masculina em procurar a UBS.

\section{DISCUSSÃO}

Observou-se, a partir dos resultados deste estudo, que para os participantes do estudo, a Avaliação das Consequências do comportamento de procurar a UBS (Atitude) não exerce influência sobre a sua Intenção Comportamental. Isso significa que mesmo estes homens fazendo uma avaliação positiva acerca da realização do comportamento, não implica necessariamente que esses tenham a Intenção de realizá-lo. No estudo de Vieira, Gomes, Borba e Costa, ${ }^{18}$ esse dado já foi demonstrado nos discursos masculinos revelando que, apesar de reconhecerem a importância destes serviços de saúde, os homens acreditavam não necessitar dos cuidados ali prestados, declarando-se saudáveis, mesmo sem conhecerem seu estado de saúde.

Nesse viés, pode-se dizer que os respondentes demonstram estar mais preocupados com os motivos que tendem a facilitar e/ou dificultar a sua decisão (Percepção de Controle) do que com as possíveis consequências que a sua decisão trará (Atitude). Entretanto, dentre os estudos realizados a partir da TAP, a Atitude das pessoas é um forte elemento que influencia a Intenção de realizar um determinado comportamento ${ }^{19-21}$.

A exemplo disso, tem-se a boa adesão que os indivíduos participantes de pesquisas na perspectiva da TAP fazem do construto Atitude, estando em quase todas as pesquisas próximo ao valor máximo da escala $\mathrm{e}$ correlacionado com a Intenção, diferente deste estudo em que apenas a média de adesão foi alta ${ }^{22-23}$.

O construto Norma Subjetiva, no presente estudo, também não apresentou correlação com a Intenção Comportamental, apesar de apresentar alta adesão pelos respondentes, contrariando os achados das diversas pesquisas realizadas pelo modelo da $\mathrm{TAP}^{22,24}$.

Por fim, o construto Percepção de Controle, que no presente estudo foi destaque para a predição do comportamento-alvo, igualmente salientou-se nos trabalhos de Hoppe, Barcellos, Vieira e Matos ${ }^{25}$ e Heath e Gifford ${ }^{26}$ em que, além de se relacionar com a Intenção Comportamental, os resultados também demonstraram alta concordância dos respondentes ao construto. Sendo assim, para os homens pesquisados, a intenção em relação ao comportamento depende estreitamente da condição de controle. Isso significa que para ter intenção de procurar a UBS é necessário que este serviço tenha vaga para atendimento médico, assim como é preciso que estes homens estejam doentes e necessitam tomar medicação.

Nesse sentido, pode-se dizer que a Intenção Comportamental dos participantes deste estudo de procurar as UBS tem baixa precisão, pois, conforme explicado por Ajzen ${ }^{14}$, para que um comportamento venha a ser realizado com sucesso é necessária a combinação das variáveis independentes (Atitude, Norma Subjetiva e Percepção de Controle) com a variável dependente 
(Intenção Comportamental), o que foi identificado parcialmente neste estudo.

Entretanto, a TAP também defende que o controle pode ser mais influente sobre o comportamentoalvo do que a opinião pessoal ou social, uma vez que Intenção Comportamental e Percepção de Controle são necessárias para o desempenho de um comportamento mantendo estreita relação, de tal maneira que o efeito da Intenção sobre o Comportamento depende da Percepção de Controle. Além disso, a Percepção de Controle pode ligar-se diretamente ao comportamento, sem necessariamente ser mediado pela Intenção, o que nos leva a crer que, segundo os resultados desta pesquisa, em casos de adoecimento ou de tomar medicação os homens provavelmente irão procurar as UBS.

\section{CONCLUSÃO}

Pelas análises foi possível evidenciar que apenas o construto Percepção de Controle se relacionou com a Intenção Comportamental. No entanto, os construtos Norma Subjetiva e Atitude não apresentaram impacto significativo sobre a Intenção dos homens de procurar a UBS. Considera-se que os participantes não se preocupam tanto com as consequências (mesmo que positivas) de procurar a UBS, nem se importam com a influência que os referentes do seu convívio social exercem para a realização deste comportamento. Tendo em vista o atual índice de morbidade e mortalidade masculina, inclusive as altas taxas de internação hospitalar desse público, as evidências apontadas pela presente pesquisa podem ser úteis para que os gestores de saúde local busquem alternativas mais eficazes a fim atrair os homens às UBS's.

Indicamos algumas limitações que, mesmo considerando as contribuições de ordem teórica e metodológica deste trabalho, podem ser discutidas. Destacamos o fato de não termos utilizado uma amostra aleatória, que foi selecionada por conveniência, podendo seus resultados serem restritos pelo tipo de atendimento que é prestado por meio das equipes de saúde do distrito sanitário pesquisado, e não pelo conhecimento genérico que fazem das UBS's. Isso pode ter gerado um tipo de resposta bastante padronizado, com pouca variabilidade e aquém do que se configura como dado representativo do contexto de Maceió (AL).

\section{REFERÊNCIAS}

1. Rocha EM, Medeiros ADL, Rodrigues KSLF, Cruz JPMC, Siqueira MFC, Farias EFNF, Lemes AG. A Política Nacional de Saúde do Homem e os Desafios de sua Implementação na Atenção Primária à Saúde. Interdisciplinar: Rev Elet Universidade [Internet]. 2016 [Acesso em: 07 out. 2017]; 15(1): 43-48. Disponível em: < http://www.univar.edu.br/revista/ index.php/interdisciplinar/article/view/476>.

2. Instituto Brasileiro De Geografia e Estatística . Diretoria de Pesquisas Coordenação de População e Indicadores Sociais. Tábua completa de mortalidade para o Brasil: breve análise da evolução da mortalidade no Brasil. 2015 [acesso em: nov. 2016]; Rio de Janeiro: IBGE. Disponível em: <ftp:/ftp. ibge.gov.br/Tabuas_Completas_de_Mortalidade/ Tabuas_Completas_de_Mortalidade_2015/tabua_ de_mortalidade_analise.pdf $>$.

3. Moreira MCN, Gomes R, Ribeiro CR. E agora o homem vem?! Estratégias de atenção à saúde dos homens. Cad. Saúde Pública [Internet]. 2016 [acesso em 2018 Maio 07]; 32(4). Disponível em: $\quad$ http://www.scielo.br/scielo.php?script $=$ sci

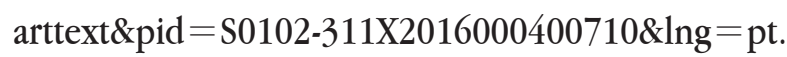
Epub 10-Maio-2016. http://dx.doi.org/10.1590/0102311X00060015.

4. Storino LP, Souza KV, Silva KL. Necessidades da saúde de homens na atenção básica. Esc Anna Nery [Internet]. 2013 [Acesso em 21 dez. 2017]; 17 (4): 638-45. Disponível em: $<$ http://saudepublica.bvs.br/ pesquisa/resource/pt/lil-697753>.

5. Albuquerque GA, Leite MF, Belém JM, Nunes JFC, Oliveira MA, Adami F. O homem na atenção básica: percepções de enfermeiros sobre as implicações do gênero na saúde. Esca Anna Nery [Internet]. 2014 [Acesso em dez. 2017]; 18(4): 607-14. Disponível em: http://www.scielo.br/scielo.php?script $=$ sci_ arttext\&pid $=$ S1414-81452014000400607\&lng =en. http://dx.doi.org/10.5935/1414-8145.20140086.

6. Souza AR, Queiroz A,M, Florêncio RMS, Portela PP, 
Fernandes JD, Pereira A. Homens nos serviços de atenção básica à saúde: repercussões da construção social das masculinidades. Rev Baiana Enferm [Internet]. Jul./set.2016 [Acesso em nov. 2017]; 30(3): 1-10. Disponível em: https://portalseer. ufba.br/index.php/enfermagem/article/view/16054. doi:http://dx.doi.org/10.18471/rbe.v30i3.16054.

7. Schraiber LB. Necessidades de saúde, políticas públicas e gênero: a perspectiva das práticas profissionais. Ciênc. saúde coletiva [Internet]. 2012 [Acesso em: 07 out. 2017]; 17(10): 2635-2644. Disponível em: <http://www.redalyc.org/articulo. oa?id $=63024360013>$.

8. Portela GZ. Atenção Primária à Saúde: um ensaio sobre conceitos aplicados aos estudos nacionais. Physis [Internet]. 2017 [Acesso em nov. 2017]; 27(2): 255-76. Disponível em: http://www.scielo. br/scielo.php?script $=$ sci_arttext\&pid $=$ S010373312017000200255\&lng=pt. http://dx.doi. org/10.1590/s0103-73312017000200005.

9. Brasil. Política Nacional de Atenção Integral à Saúde do Homem: princípios e diretrizes, de novembro de 2008.Ministério da Saúde, Brasília, DF, 2009.

10. Moura EC, Santos W, Neves ACM, Gomes R, Schwarz E. Atenção à saúde dos homens no âmbito da Estratégia Saúde da Família. Ciênc. saúde coletiva [Internet]. 2014 [Acesso em dez. 2017]; 19(2): 42938. Disponível em: Available from: http://www.scielo. br/scielo.php?script $=$ sci_arttext\&pid $=$ S1413$81232014000200429 \& \operatorname{lng}=$ en. $\quad$ http://dx.doi. org/10.1590/1413-81232014192.05802013.

11. Separavich MA, Canesqui AM. Saúde do homem e masculinidades na Política Nacional de Atenção Integral àSaúde do Homem: uma revisão bibliográfica. Saúde Soc [Internet]. 2013 [Acesso em out. 2017]; 22(2): 415-428. Disponível em: http://www.scielo. br/scielo.php?script $=$ sci_arttext\&pid $=$ S010412902013000200013\&lng=pt\&tlng=pt. doi:http:// dx.doi.org/10.1590/S0104-12902013000200013.

12. Moutinho K, Roazzy A. As Teorias da Ação Racional e da Ação Planejada: relações entre intenções e comportamentos.Aval.psicol. [Internet]. 2010 [Acesso em: 07 out. 2017; 9(2): 279-287. Disponível em: $<$ http://pepsic.bvsalud.org/scielophp?script $=$ sci arttext\&pid $=$ S1677-04712010000200012\&lng $=$ pt $\&$ tlng $=$ pt. $>$.

13. Fishbein M, Ajzen I. Belief, attitude, intention, andbehavior: anintroductiontotheoryandresearch. Reading, MA: Addison-Wesley; 1975.

14. Ajzen I. The theory of planned behavior. Organizational Behavior and Human Decision Process. 1991;50(2):179-211. doi:https://doi. org/10.1016/0749-5978(91)90020-T.

15. Ajzen I. Behavioral Interventions: Design andevaluationguidedbythe theory of planned behavior. In: Mark M M, Donaldson S I, Campbel B. (Ed.). Social psychology and evaluation. New York: Guilford Press; 2011. p. 72-100.

16. Bardin L. Análise de conteúdo. São Paulo, SP: Edições; 70, 2011.

17. Hair JRJF, Black WC, Babin BJ, Anderson RE, Tatham RL. Análise multivariada de dados. Porto Alegre: Bookman; 2005.

18. Vieira KLD, Gomes VLO, Borba MR, Costa CFS. Atendimento da população masculina em unidade básica saúde da família: motivos para a (não) procura. Esc Anna Nery [Internet]. 2013 [Acesso em: $21 \mathrm{dez}$. 2017]; 17(1):120-7. Disponível em: <http://www. portalsaude.ufms.br/posts/view/214?s $=2 \#>$.

19. Chinazzo IR, Câmara SG, Frantz DG. Comportamento sexual de risco em jovens: aspectos cognitivos e emocionais. Psico-USF, Bragança Paulista [Internet]. jan./abril, 2014 [Acesso em: nov. 2017];19(1):1-12. Disponível em: <http://www. scielo.brscielophp?script $=$ sci_arttext\&pid $=S 1413$ 82712014000100002\&lng=pt\&nrm=iso > . http:// dx.doi.org/10.1590/S1413-82712014000100002.

20. Mendes FL, Batista JO, Cacho ANB, Soares ALV. Aplicativos Móveis e Turismo: um estudo quantitativo 
aplicando a Teoria do Comportamento Planejado. Revista Rosa dos Ventos - Turismo e Hospitalidade [Internet]. abr/jun, 2017 [Acesso em: 21 dez. 2017]; 9(2):179-99. Disponível em: http:/ucs.br/etc/revistas/ index.php/rosadosventos/article/download/4787/pdf.

21. Torquato R, Franco CMA, Bianchi A. SeatBelt Use Intention among Brazilian Undergraduate Students. Revista Colombiana de Psicología, Bogotá [Internet]. july/dec. 2012 [Acesso em: 07 out. 2017]; 21(2): 253-63. Disponível em: http://www.scielo.org. co/scielo.php?script $=$ sci_arttext\&pid $=$ S0121$54692012000200005 \& \operatorname{lng}=$ en\&nrm $=$ iso.

22. Cabral TRP. Intenção do uso de preservativo das mulheres de João Pessoa:aspectos psicológicos e sociais. 2014. 136f. [Dissertação] [Internet] Universidade Federal da Paraíba, João Pessoa, 2014. [Acesso em: nov. 2017]. Disponível em: http://tede. biblioteca.ufpb.br/handle/tede/7562.

23. Maciel MG, Veiga RT. Intenção de mudança de comportamento em adolescentes para a prática de atividades físicas de lazer. Rev. bras. Educ. Fís. Esporte, São Paulo [Internet]. out./dez. 2012 [Acesso em: 21 dez. 2017]; 26(4): 705-16. Disponível em: $\quad$ www.scielo.br/scielo.php?script $=$ sci arttext\&pid $=$ S1807-55092012000400014.

24. Souza GFM, Borges FRF, Lopes ALM, Muniz RM. Propagação de mensagens na internet: Teoria do comportamento planejado. Pretexto [Internet]. jul/set. 2012 [Acesso em: 21 dez. 2017]; 13(3): 1127. Disponível em: http://www.fumec.br/revistas/ pretexto/article/view/1258.

25. Hoppe A, Barcellos MD, Vieira LM, Matos CA. Comportamento do consumidor de produtos orgânicos: uma aplicação da teoria do comportamento planejado. Rev Base (Administração e Contabilidade) da UNISINOS [Internet]. 2012 [Acesso em: 06 de out. 2017]; 9(2): 174-88. Disponível em: http://www. redalyc.org/articulo.oa?id=87880103.

26. Heath Y, Gifford R. Extending The Theory Of Planned Behavior: predicting the use of public transportation. J Appl Soc Psychol [Internet]. 2002 [Acesso em: dez. 2017]; 32(10): 2154-89. Disponível em : https://onlinelibrary.wiley.com/doi/ abs/10.1111j.1559-1816.2002.tb02068.x

Recebido em: 22/12/2017

Aceito em: 25/05/2018 